\title{
BMJ Open Improving the quality of routine maternal and newborn data captured in primary health facilities in Gombe State, Northeastern Nigeria: a before- and-after study
}

Antoinette Alas Bhattacharya (D) , ${ }^{1}$ Elizabeth Allen, ${ }^{2}$ Nasir Umar, ${ }^{1}$ Ahmed Audu, ${ }^{3}$ Habila Felix, ${ }^{3}$ Joanna Schellenberg, ${ }^{1}$ Tanya Marchant ${ }^{1}$

To cite: Bhattacharya AA, Allen $\mathrm{E}$, Umar N, et al. Improving the quality of routine maternal and newborn data captured in primary health facilities in Gombe State, Northeastern Nigeria: a beforeand-after study. BMJ Open 2020;10:e038174. doi:10.1136/ bmjopen-2020-038174

- Prepublication history and additional materials for this paper is available online. To view these files, please visit the journal online (http://dx.doi. org/10.1136/bmjopen-2020038174).

Received 01 March 2020 Revised 15 0ctober 2020 Accepted 05 November 2020

Check for updates

(c) Author(s) (or their employer(s)) 2020. Re-use permitted under CC BY. Published by BMJ.

${ }^{1}$ Department of Disease Control, London School of Hygiene \& Tropical Medicine Faculty of Infectious and Tropical Diseases, London, UK

${ }^{2}$ Department of Medical Statistics, London School of Hygiene \& Tropical Medicine Faculty of Epidemiology and Population Health, London, UK ${ }^{3}$ Gombe State Primary Health Care Development Agency, Gombe, Nigeria

\section{Correspondence to} Antoinette Alas Bhattacharya; antoinette.bhattacharya@Ishtm. ac.uk

\section{ABSTRACT}

Objectives Primary objective: to assess nine data quality metrics for 14 maternal and newborn health data elements, following implementation of an integrated, district-focused data quality intervention. Secondary objective: to consider whether assessing the data quality metrics beyond completeness and accuracy of facility reporting offered new insight into reviewing routine data quality.

Design Before-and-after study design.

Setting Primary health facilities in Gombe State, Northeastern Nigeria.

Participants Monitoring and evaluation officers and maternal, newborn and child health coordinators for state-level and all 11 local government areas (districtequivalent) overseeing 492 primary care facilities offering maternal and newborn care services.

Intervention Between April 2017 and December 2018, we implemented an integrated data quality intervention which included: introduction of job aids and regular selfassessment of data quality, peer-review and feedback, learning workshops, work planning for improvement, and ongoing support through social media.

Outcome measures 9 metrics for the data quality dimensions of completeness and timeliness, internal consistency of reported data, and external consistency. Results The data quality intervention was associated with improvements in seven of nine data quality metrics assessed including availability and timeliness of reporting, completeness of data elements, accuracy of facility reporting, consistency between related data elements, and frequency of outliers reported. Improvement differed by data element type, with content of care and commodity-related data improving more than contact-related data. Increases in the consistency between related data elements demonstrated improved internal consistency within and across facility documentation. Conclusions An integrated district-focused data quality intervention-including regular self-assessment of data quality, peer-review and feedback, learning workshops, work planning for improvement, and ongoing support through social media—can increase the completeness, accuracy and internal consistency of facility-based routine data.

\section{Strengths and limitations of this study}

- We extended the evidence on integrating data quality interventions within existing systems to improve the quality of facility-based data for monitoring and planning.

- We demonstrated the value of an integrated districtfocused data quality intervention to include regular self-assessments of data quality, peer-review and feedback, work planning for improvement, and ongoing support through social media.

- We assessed the usefulness of the WHO's catalogue of data quality metrics to measure and monitor the quality of routine facility data, as data quality studies primarily review completeness and accuracy of facility reporting.

- Without a concurrent comparison group, our beforeand-after analyses cannot eliminate the effects of concurrent events and activities on data quality metrics.

\section{INTRODUCTION}

Routine health information systems provide essential data for governments and stakeholders to make decisions for managing performance and optimising service delivery. $^{1-3}$ Routine health information systems, which include facility-based data, have the potential to provide disaggregated statistics important for understanding disparities and inequities in the provision of quality services and related health outcomes. ${ }^{45}$

Effective use of routine health data is dependent, in part, on the quality of data. ${ }^{146-9}$ Studies assessing the quality of routine health data have shown persistent challenges in incomplete and untimely reporting, incomplete indicator-level data, inaccurate facility reporting, and imprecise target population estimates for coverage. ${ }^{10-13}$ Further, studies 
have noted considerably poorer data quality at the facilitylevel than at district, state and national-levels, citing challenges in accurately capturing data as well as in tallying and summarising service data for monthly reporting. ${ }^{14-16}$

Improving the quality of routine data is a priority given the potential of routine data to contribute to effective programme monitoring and performance management. Efforts to improve the quality of routine data have included data quality checking with feedback as a low-resource and low-cost activity for strengthening data quality, particularly when implemented as a routine activity. Data quality checking with feedback have been shown to improve completeness, timeliness and accuracy of facility reporting. ${ }^{4} 7101718$ Knowledge transfer activities, such as mentoring, training or workshops, have offered an opportunity to receive additional skills building in data quality checking or data use. ${ }^{6} 8$ 19-28 Knowledge sharing activities such as data review meetings and dashboards have brought together different health system-levels or peers within a health system level and include understanding the relative performance of teams on service coverage indicators or on dimensions of data quality. ${ }^{6} 82022$ 29-31 The introduction of technology, either software such as District Health Information Software V.2 (DHIS2) or a device to enhance data collection such as a tablet, has demonstrated improvements in completeness, timeliness and error detection. However, these technology-based initiatives often required supervision, monitoring and feedback to ensure errors were resolved and that data entered were accurate and consistent. $^{7273233}$ Activities which aligned with user priorities and were integrated within existing government systems were perceived to be advantageous as well as more likely to be adopted and adapted. ${ }^{20}$

Within the context of routine health information systems, the WHO has characterised routine data quality into four broad dimensions: completeness and timeliness; internal consistency; external consistency and external comparisons. ${ }^{34}$ While the above-mentioned data quality interventions have demonstrated increases in completeness and accuracy of facility reporting, ${ }^{483536}$ there are few peer-reviewed studies that quantitatively assessed changes in data quality metrics beyond this. ${ }^{39} 37$

In this study, our primary objective was to measure the changes in data quality metrics before and after the introduction of an integrated district-focused intervention in Northeastern Nigeria for routine facility data captured in primary health facilities. A secondary objective was to determine the extent to which expanding data quality metrics beyond completeness and accuracy of facility reporting offered new insight into reviewing data quality.

\section{METHODS}

\section{Study design}

This was a before-and-after study design for a data quality intervention in all 11 local government areas (LGA, district-equivalent) of Gombe State, Northeastern
Nigeria. We present results for the state ( $\mathrm{n}=492$ facilities), comparing the 21-month period before the intervention, July 2015-March 2017, with the 21-month period after introducing the intervention, April 2017-December 2018.

\section{Patient and public involvement}

Patients or the public were not involved in the design, conduct, reporting or dissemination of the research described here.

\section{Study setting}

Gombe State is located in Northeastern Nigeria, a region with high maternal and newborn mortality at 1549 per 100000 live births and 35 per 1000 live births, respectively. ${ }^{38}$ With an estimated population of 2.9 million, Gombe is predominantly rural and $35 \%$ of the women have some primary school education. ${ }^{40}{ }^{41}$ Most women access maternity care through public facilities. Seventy-two per cent of women reported at least one antenatal care visit during their last pregnancy and $28 \%$ gave birth in a health facility. ${ }^{39}$ In 2018 , over $70 \%$ of facility deliveries took place in rural primary health facilities. ${ }^{42}$

Under Nigeria's national policy of Primary Health Care Under One Roof, the Gombe State Primary Health Care Development Agency oversees the administration and service delivery for primary health facilities across 11 LGA; each LGA has 10-11 political wards (114 wards, total). ${ }^{43}$ LGA monitoring and evaluation officers are responsible for community-level and facility-level data collection, validation and reporting to the state office. LGA maternal, neonatal and child health coordinators support the supervision and implementation of services for women and children.

During the intervention period, Gombe State had 492 primary health facilities providing antenatal and childbirth services. As in other states in Nigeria, Gombe facility staff generally completed 13 paper-based registers to document the services they provide (Nigeria health management information system, V.2013). Every month, a subset of data in these registers were tallied and summarised in a paper-based report and sent to the LGA health office to be entered into DHIS2.

\section{Data quality intervention}

The routine data quality intervention period spanned 21 months, from April 2017 through December 2018. Following a situational analysis and literature review, development of the intervention took place in consultation with the Gombe State Primary Health Care Development Agency. The design was predicated on three key issues. Interventions that include data quality assessments and feedback have demonstrated improvement in completeness, timeliness and consistency. ${ }^{4} 6192022$ 23 26-28 Monitoring and evaluation officers and programme coordinators in the state were expected to engage with data from facilities to support improvement of service delivery; however, their interactions were not optimally structured 
to ensure efficient and complementary engagement with facility data. Further, the recent publication of the WHO data quality review toolkit in early 2017 provided clarity on assessing the data quality dimensions and metrics for facility data; however, the Gombe district staff required further support and job aids to administer the data quality reviews as recommended in the WHO guidance. ${ }^{45}$

Thus, the intervention emphasised the partnership between the LGA monitoring and evaluation officer and the LGA maternal, newborn and child health programme coordinator to underscore the link between the quality and use of routine data. ${ }^{8}$ The intervention was designed to facilitate existing LGA-level supervision responsibilities. It leveraged scheduling of ongoing activities to minimise cost, added job aids based on the WHO data quality review toolkit, and defined performance standards to provide structure to existing data quality checking duties and to target feedback to facilities.

The intervention included the following activities: (i) data quality learning workshops to present data quality selfassessment findings and develop work plans for improvement; (ii) defining data quality performance standards and milestones for completeness, timeliness and consistency; (iii) introduction of job aids to self-assess data quality according to the WHO data quality metrics; (iv) monthly state-level and LGA-level data quality summary reports; (v) intentional practice on providing constructive feedback to peers and low-performing facilities to promote a positive culture of data use and (vi) ongoing engagement on data quality issues through governmentapproved communication channels, including the social media application WhatsApp.

Twenty-six main attendees participated in the workshops and ongoing communication in-between workshops. These included two participants from each of the 11 LGAs, a monitoring and evaluation officer and the maternal, newborn and child health programme coordinator. At the state-level, four officials participated: the director of the Gombe State Planning, Research, and Statistics Department, the state monitoring and evaluation officer, the state health management information systems officer, and the state maternal, newborn and child health coordinator.

Four data quality learning workshops took place every 6-9 months. The 2-day workshops included the introduction of job aids and practical sessions to strengthen data quality checking skills, the presentation of the state's and each LGA's self-assessment of data quality, and the development of 6-month work plans to improve the quality of facility-based routine data. Using materials designed for postgraduate learning and teaching, there was intentional practice on how to provide constructive feedback to peers and facilities to promote a positive culture of information use. ${ }^{46}$

At each workshop, a major theme emerged during the work planning sessions (figure 1). For example, at the first workshop, participants were concerned with inconsistencies observed between the paper-based facility

\begin{tabular}{|l|}
\hline Workshop 1 \\
\hline Learning and practice: \\
\hline - Data quality dimensions, metrics \\
- Job aids for self-assessment \\
- Work planning for improvement \\
Participant presentation: \\
Present findings for select data \\
quality metrics (completeness of \\
reporting and agreement between \\
facility register data and reports) \\
Main outputs from work planning: \\
- Finalise standards and \\
milestones for data quality \\
reporting by LGAs \\
- Revitalise monthly LGA validation \\
committee \\
- Revitalise WhatsApp group for \\
ongoing communication
\end{tabular}

\begin{tabular}{|l|}
\hline Workshop 2 \\
\hline Learning and practice: \\
- Quarterly self-assessment \\
practice with job aids \\
- Interpreting and visualising self- \\
assessment findings \\
Participant presentation: \\
Present quarterly self-assessment \\
findings for peer review and \\
feedback \\
Main outputs from work planning: \\
- Adjust data quality metric \\
calculations to exclude inactive \\
facilities and indicators in DHIS2 \\
- Improve completeness of data \\
elements \\
- Identify facilities with low data \\
quality metrics and call/visit \\
them to problem-solve
\end{tabular}

\begin{tabular}{|l|}
\hline Workshop 3 \\
\hline Preparation before workshop: \\
\hline - Quarterly data quality self- \\
assessment, January-March 2018 \\
- Investigate reasons for facilities' \\
higher/lower data quality metrics \\
Learning and Practice: \\
- Interpreting and visualising self- \\
assessment findings \\
- Comparing LGA-level findings \\
with performance of Gombe \\
State and with neighbouring LGA \\
- Providing positive and \\
constructive feedback \\
Participant presentation: \\
Joint presentation (with \\
neighbouring LGA) of quarterly \\
review findings \\
Main outputs from work planning: \\
- Clean up inactive facilities and \\
indicators in DHIS2 \\
- Identify facilities with low data \\
quality metrics and call/visit them \\
to problem-solve \\
- Practice positive feedback
\end{tabular}

Workshop 4

Preparation before workshop:

- Bi-annual data quality selfassessment, May-October 2018

- Investigate reasons for facilities' higher/lower data quality metrics

Learning and Practice:

- Elements of an effective presentation and feedback

Participant presentation:

- Bi-annual data quality findings and priorities for next 6 months

- Demonstrating positive feedback to presenters

Main outputs from work planning: - Improve consistency between related data elements, focus feedback on facilities with higher inconsistency between first antenatal care visits and related services

- Provide faster, real-time feedback to facilities

Figure 1 Data quality learning workshops in Gombe State, April 2017-December 2018. DHIS2, District Health Information Software V.2; LGA, local government areas. 
registers and the facility's monthly summary reports. Activities enacted from the work planning session were to revitalise dormant strategies previously set up to address programme monitoring and evaluation activities: (i) LGA data validation committee meetings, where facilities bring their registers for verification against their submitted monthly facility report and (ii) a social media WhatsApp group of LGA actors and facilities. The LGA teams posted pictures and comments on these facility interactions on the WhatsApp group for encouragement and accountability.

After the first workshop, the Gombe State monitoring and evaluation officer disseminated monthly state-level and LGA-level data quality summary reports. LGAs were assessed according to the WHO data quality metrics and recommendations for improvement were offered. Initially, this activity was designed for the external workshop facilitators to compose and disseminate, while building the capacity of the state officer to take on this task over time.

\section{Outcomes}

Using the WHO data quality review toolkit for routine facility data, we assessed nine metrics across the three data quality dimensions of completeness and timeliness; internal consistency; and external consistency. ${ }^{45}$ The data sources and analyses for each data quality metric are described in the following section. Online supplemental table S1 provides additional information on each data quality metric assessed and the data sources reviewed.

\section{Data analysis and data sources}

Three data sources were used to assess the routine data quality metrics, described later: facility-reported data in DHIS2, external facility surveys, and external household surveys.

DHIS2 contained monthly reports for the 492 primary facilities providing antenatal care, childbirth, and postnatal care services. Monthly aggregated DHIS2 data for July 2015-December 2018 were downloaded at one time and included 14 maternal and newborn health-related data elements.

These data were used to assess availability of facility reporting; timeliness of facility reporting; completeness of all 14 priority maternal and newborn health data elements, per monthly facility report; completeness of data element; presence of moderate and extreme outliers; consistency of indicator values over time and consistency between related data elements.

To assess completeness of facility reporting, we compared the proportions of facilities submitting reports before the intervention and during the intervention. To assess timeliness of facility reporting, the numerator for completeness of facility reporting was further limited to facilities that submitted the reports by the given timelines. To assess both completeness of data-related metrics, we compared the proportions of facility reports where a value was present for the relevant data. To compare the proportions of the preintervention and intervention periods, we used linear mixed models to account for the clustering of facility measurements within facilities and the clustering of facilities within districts. $\mathrm{P}$ values for these metrics were computed from the Wald test within the mixed models. In these models, we also adjusted for potential confounders such as total client volume and time. $^{46}$

We calculated the intraclass correlation coefficient (ICC) as a measure of agreement for the WHO metric assessing consistency between related data elements. ICC values range between 0 and 1 , where values approaching 1 represented greater agreement between two related data elements. As the intervention worked through districtlevel staff to improve data quality across 492 primary health facilities, a paired t-test was carried out to compare preintervention and intervention periods matching ICCs at the district-level.

In 2016 and 2018, facility-level surveys were conducted in 97 primary facilities across Gombe to assess their readiness to provide maternal and newborn health services. The two surveys represented the approximate midpoints of the preintervention and intervention period. The selected facilities were a state-wide random sample drawn from all primary health facilities. Detailed methods are reported elsewhere. ${ }^{47}$ The facility survey protocol was similar to a Service Availability and Readiness Assessment and also included data extraction from the facility's paper-based antenatal and postnatal care register and the labour and delivery register (Nigeria health management information system, V.2013). ${ }^{48}$ A trained third party data collection team tallied and recorded the register data for each month of the 6-month periods immediately prior to the survey: January-June 2016 and February-July 2018.

These data were used to assess the accuracy of facility reporting (also referred to as data accuracy, data verification or concordance in peer-reviewed literature). We compared the facilities' paper-based registers data with the facilities' monthly reported data in DHIS2. As with the consistency between related data elements metric, we calculated the ICC as a measure of agreement and carried out a paired t-test for the preintervention and intervention periods matching ICCs at the district level. ICC values approaching 1 represented greater agreement.

In 2016 and 2018, household-level surveys were conducted in the enumeration areas of the abovementioned 97 primary facilities to assess access to and quality of maternal and newborn services. ${ }^{47}$ A total of 79 enumeration areas were surveyed since some enumeration areas were served by more than one facility. All households in each enumeration area were surveyed. The household surveys included a mother's module which asked all women who reported a birth in the last year a detailed set of questions about their contact with health services across the continuum of care from pregnancy to postnatal care. Informed consent was obtained at the community leadership-level and at the individual-level 
for each respondent. All invited participants agreed to be interviewed.

These data were used for external consistency during the preintervention and intervention periods. We compared coverage estimates from household surveys to those from the 97 matching facilities in DHIS2. We compared the same recall period for the surveys and the DHIS2. The DHIS2 data are considered consistent if they fall within the CIs of the external household survey estimates. Calculations of point estimates and their 95\% CIs were done using the svyset Stata 14.2 command to adjust for clustering. We chose the highest-order clustering level to provide the most conservative CI estimates. ${ }^{49}$

\section{RESULTS}

An integrated district-focused data quality intervention was implemented across 11 LGAs overseeing 492 primary health facilities providing maternal and newborn care services. Below, we present the results for nine data quality metrics.

\section{Completeness and timeliness}

Table 1 summarises the completeness and timeliness of reporting at the facility-level and indicator-level. At the facility-level, the availability of monthly facility reports improved from $72 \%$ to $82 \%(\mathrm{p}<0.001)$ and timeliness of submitting the reports increased from $60 \%$ to $72 \%$ $(p<0.001)$. The proportion of facility-months where all 14 priority maternal and newborn health data elements contained a value within the monthly report increased from $62 \%$ to $68 \%(\mathrm{p}<0.001)$.

At the indicator-level, 7 of 14 data elements assessed improved in completeness compared with the preintervention period. Indicator-level completeness did not

Table 1 Facility-level and indicator-level completeness and timeliness, Gombe State ( $n=492$ facilities)

\begin{tabular}{lll}
$\begin{array}{l}\text { Preintervention } \\
\text { July 2015-March }\end{array}$ & & Intervention \\
2017 & $\begin{array}{l}\text { April 2017- } \\
\text { December } 2018\end{array}$ \\
\hline$\%(95 \% \mathrm{Cl})$ & $\%(95 \% \mathrm{Cl})$ \\
\hline
\end{tabular}

Facility-level

Availability of monthly facility reports

72 (69 to 74$)$

82 (80 to 84$)$

Timeliness of monthly facility reports

60 (57 to 62$)$

$72(70$ to 74$)$

Completeness of all 14 priority maternal and newborn health data elements, per monthly facility report

$62(60$ to 63$)$

68 (66 to 70$)$

\section{Indicator-level}

For every 100 facilities that submitted a monthly facility report, the percentage of facilities reporting a value for the following services

$\begin{array}{lcc}\text { First antenatal care visits } & 76 \text { (67 to } 85) & 77(70 \text { to } 84) \\ \text { Total antenatal care visits } & 100 \text { (99 to } 100) & 100(100 \text { to } 100) \\ \text { Facility deliveries } & 68(59 \text { to } 77) & 67(60 \text { to } 74)\end{array}$

For every 100 facilities that reported a value for first antenatal care visit, the percentage of facilities reporting a value for the following services

\begin{tabular}{lll} 
Antenatal care anaemia testing & 28 (16 to 39$)$ & 36 (24 to 49$)$ \\
Antenatal care syphilis testing & 42 (23 to 61$)$ & 29 (23 to 35$)^{\star}$ \\
\hline Iron-folic acid supplementation & $80(75$ to 84$)$ & 89 (85 to 92$)$ \\
At least one dose administered of intermittent preventive treatment of malaria & 45 (34 to 56$)$ & 56 (49 to 62$)$ \\
At least one dose administered of tetanus toxoid vaccine & 90 (86 to 93$)$ & 89 (86 to 91$)$
\end{tabular}

For every 100 facilities that reported a value for a facility delivery, the percentage of facilities reporting a value for the following services

\begin{tabular}{|lll|}
\hline Delivery by skilled birth attendant & 43 (25 to 61$)$ & 86 (81 to 91$)$ \\
\hline Live birth or stillbirth & 90 (86 to 95$)$ & 96 (94 to 97$)$ \\
\hline Baby weighed at birth & 89 (83 to 95$)$ & 95 (94 to 97$)$ \\
\hline Oral polio vaccine given at birth & 79 (70 to 87$)$ & 86 (82 to 90$)$ \\
\hline Early postpartum-postnatal care within 3 days of birth & 45 (38 to 53) & 55 (46 to 64) \\
\hline BCG vaccine given during postnatal care period & 79 (71 to 88) & 81 (77 to 86) \\
\hline
\end{tabular}

*During the intervention period, commodities for antenatal care syphilis testing were redistributed and restricted to 57 facilities. For these 57 facilities, completeness of data for antenatal care syphilis testing increased from $48 \%$ (95\% Cl 28 to 68 ) to $77 \%$ (95\% Cl 69 to 86 ). 
Table 2 Internal consistency: consistency between data elements with a predictable relationship ( $\mathrm{n}=492$ facilities)

\begin{tabular}{|c|c|c|c|}
\hline Data elements with a predictable relationship & $\begin{array}{l}\text { ICC, Preintervention } \\
(95 \% \text { Cl) }\end{array}$ & $\begin{array}{l}\text { ICC, Intervention } \\
95 \% \mathrm{CI}\end{array}$ & $P$ value \\
\hline \multicolumn{4}{|l|}{ Concurrent tallying within and across facility documentation } \\
\hline Total postnatal care visits=sum (postnatal care visit categories) & $0.46(0.36$ to 0.57$)$ & $0.76(0.66$ to 0.85$)$ & $<0.001$ \\
\hline \multicolumn{4}{|l|}{ Relationship between first antenatal care visits and } \\
\hline At least one dose of intermittent preventive treatment of malaria & $0.33(0.17$ to 0.48$)$ & $0.43(0.32$ to 0.54$)$ & 0.129 \\
\hline At least one dose of tetanus-toxoid vaccine & $0.67(0.53$ to 0.80$)$ & $0.73(0.65$ to 0.82$)$ & 0.167 \\
\hline \multicolumn{4}{|l|}{ Relationship between total antenatal care visits and } \\
\hline Iron-folic acid supplementation & $0.92(0.89$ to 0.95$)$ & $0.97(0.95$ to 0.99$)$ & 0.005 \\
\hline Oral polio vaccine given at birth & $0.48(0.32$ to 0.63$)$ & $0.72(0.65$ to 0.79$)$ & 0.007 \\
\hline Early postpartum-postnatal care within 3 days of birth & $0.09(0.06$ to 0.13$)$ & $0.19(0.13$ to 0.24$)$ & $<0.001$ \\
\hline BCG vaccine given during postnatal period & $0.56(0.40$ to 0.72$)$ & $0.67(0.57$ to 0.76$)$ & 0.147 \\
\hline
\end{tabular}

ICC values range from 0 to 1 , with values approaching 1 representing greater agreement.

ICC, intraclass correlation coefficient.

change for contact indicators such as first antenatal care visits, total antenatal care visits and facility deliveries.

\section{Internal consistency: consistency between related data elements}

To assess the consistency between related data elements with a predictable relationship, two types of relationships were reviewed (table 2). The first type of relationship assessed concurrent tallying across different data elements within and across facility registers. For example (i) normal deliveries+caesarean deliveries+assisted deliveries=live births+still births and (ii) total postpartum visits reported=sum of the postnatal visit categories reported. For Gombe State, the ICC of delivery types (normal, caesarean, assisted) to birth types (live births, still births) improved from 0.83 (95\% CI 0.73 to 0.94 ) to 0.95 (95\% CI 0.93 to 0.97$)$. Similar patterns of improvement were noted for postnatal visit tallying from an ICC of 0.46 (95\% CI 0.36 to 0.57 ) to 0.76 (95\% CI 0.66 to $0.85)$.

The second type of relationship assessed was a service provision compared with a contact indicator (eg, the number of antenatal care syphilis testing done compared with antenatal care first visits, the number of babies weighed at birth compared with the number of facility deliveries). During the preintervention period, 1 of the 10 relationships reflected high consistency: ironfolic acid supplementation. During the implementation period, 7 of the 10 relationships reflected improved consistency.
Internal consistency: accuracy of facility reporting

Comparing the facilities' registers with their submitted monthly reports, accuracy of facility reporting (data accuracy) had improved for 6 of 7 indicators, reflecting greater agreement during the intervention period (table 3 ). For total antenatal care visits, there was considerable variation between districts during the preintervention and intervention periods, with an ICC of $0.62(95 \%$ CI 0.41 to 0.83 ) and 0.86 (95\% CI 0.72 to 0.99 ), respectively.

\section{Internal consistency: outliers and consistency over time}

Online supplemental tables S2 and S3 summarise the presence of outliers and the consistency over time for the 14 maternal and newborn data elements. The frequency of months when outliers were reported decreased during the intervention period. However, 11 moderate outliers were reported during the intervention period compared with eight moderate outliers and two extreme outliers reported during the preintervention period. All 11 outliers reported during the intervention period occurred in May 2018 during a health worker strike. Of the 14 data elements assessed, 6 data elements were inconsistent over time due to reported increases in services when comparing the final year of the intervention 2018 to the mean value of the last 3 years and when comparing the preintervention and intervention periods. 
Table 3 Accuracy of facility reporting: comparison of paper-based facility records and facility monthly reports in District Health Information Software 2 ( $n=97$ facilities)

\begin{tabular}{|c|c|c|c|}
\hline Data element & $\begin{array}{l}\text { ICC, Preintervention } \\
(95 \% \mathrm{Cl})\end{array}$ & $\begin{array}{l}\text { ICC, Intervention } \\
(95 \% \mathrm{Cl})\end{array}$ & P value \\
\hline First antenatal care visits & $0.57(0.40$ to 0.75$)$ & $0.88(0.83$ to 0.93$)$ & 0.004 \\
\hline Antenatal care syphilis testing & $0.08(-0.02$ to 0.18$)$ & $0.59(0.42$ to 0.76$)$ & $<0.001$ \\
\hline Facility deliveries & $0.48(0.26$ to 0.70$)$ & 0.82 (0.69 to 0.94$)$ & 0.019 \\
\hline Total postnatal care visits & $0.15(0.02$ to 0.29$)$ & $0.58(0.38$ to 0.78$)$ & 0.001 \\
\hline Early postpartum-postnatal care within 3 days of birth & 0.21 (0.01 to 0.41$)$ & $0.55(0.31$ to 0.80$)$ & 0.020 \\
\hline
\end{tabular}

ICC values range from 0 to 1 , with values approaching 1 representing greater agreement.

ICC, intraclass correlation coefficient.

\section{External consistency: agreement between facility summary reports in DHIS2 and household surveys}

Figure 2 summarises external consistency, which is the agreement between facility-based routine data in DHIS2 compared with household-level surveys in the enumeration areas of these facilities. Other than the indicator for early postpartum-postnatal care, there was no agreement nor any consistent pattern of agreement between facilitybased routine data and the household surveys. DHIS2 data underestimated compared with the household survey for at least one dose of intermittent preventative therapy for malaria in pregnancy and at least one dose of tetanus toxoid. DHIS2 data overestimated compared with the household survey for baby weighed at birth, oral polio vaccine given at birth and BCG given during postnatal period. For antenatal care anaemia testing, facility-based

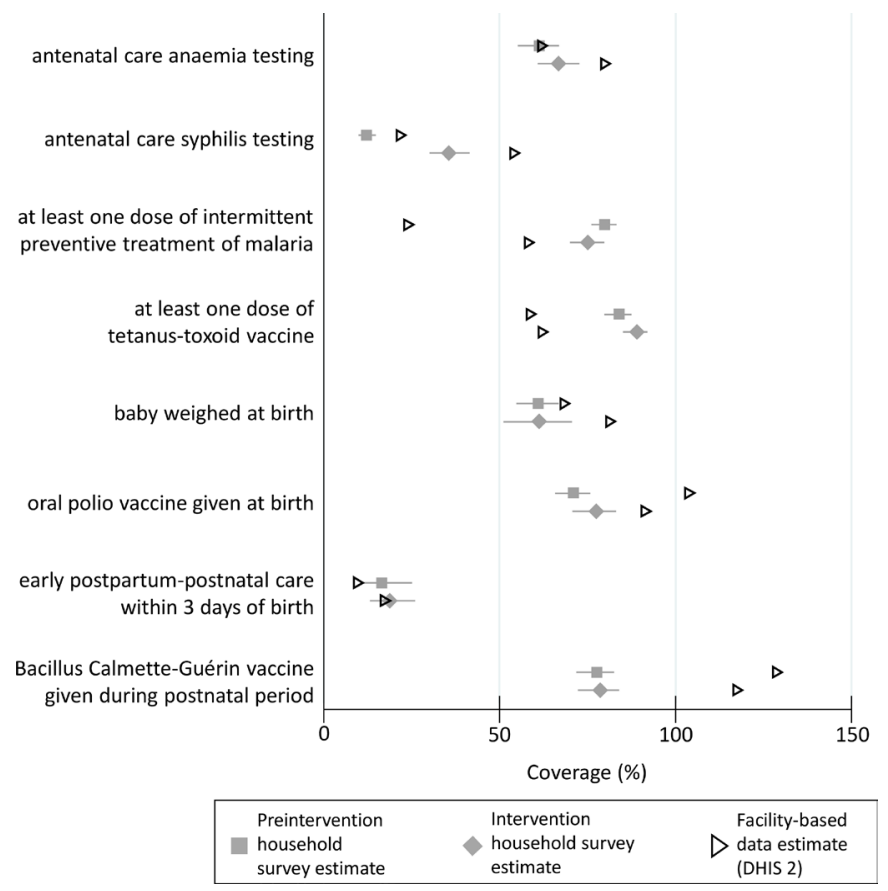

Figure 2 External consistency: comparison of householdlevel survey and facility summary reports in District Health Information Software 2 ( $\mathrm{n}=97$ facilities). estimates were within the household survey estimate CI but overestimated compared with the household survey during the intervention period.

\section{DISCUSSION}

Facility-based routine data are an important source for monitoring, performance management and planning. ${ }^{9}$ Our study found that an integrated district-focused data quality intervention-which included regular selfassessment of data quality, peer-review and feedback, learning workshops, work planning for improvement, monthly data quality reports, and ongoing support through social media-was associated with improvements across most WHO data quality metrics. There were differences in data quality improvement by data element type. ${ }^{50}$ Data related to content of care or the provision of commodities, such as syphilis testing and intermittent preventative therapy for malaria, improved more across data quality metrics compared with contact indicators. Contact indicators had relatively higher data quality metrics before the intervention, such as first antenatal care visits and facility deliveries. Contact indicators such as first antenatal care visits, fourth antenatal care visits and facility deliveries are generally well-defined events to document, have been key denominators for local programme planning, and have been prioritised for monitoring progress in previous global initiatives including the Millennium Development Goals and Countdown to $2015 .^{50-52}$

This was an integrated data quality intervention designed to facilitate existing state-level and district-level data quality checking responsibilities and emphasise the partnership between the monitoring and evaluation officers and the maternal, newborn and child health programme coordinators to expand local access to the DHIS2 data, use the data and problem solve. ${ }^{9}$ Incorporating regular data quality reviews and feedback within supervision is an acknowledged low-cost and effective activity for improving completeness and consistency. ${ }^{6} \quad 8 \quad 17 \quad 18 \quad 53$ Leveraging existing staff and meeting schedules as well as adding structure to existing data-related responsibilities further 
promotes the feasibility of this intervention in similar settings. The evolution of the intervention through the work planning sessions prompted local solutions defined by the participants as feasible and within their resources to implement. In particular, the participants' decision to revitalise the data validation committees during the first workshop engaged the facilities early on to ensure the facility's register counts matched the facility's monthly report aggregate value. This early engagement with facilities could have contributed to the increased ICCs for accuracy of facility reporting observed during the intervention period (as shown in table 3). A formative phase of the intervention might have captured activities such as the data validation committee as a predefined intervention activity. Including a formative phase should be a consideration for future implementation.

Our findings aligned with previous studies reporting improvements in completeness, timeliness and accuracy of facility reporting after intervention. However, this study's relative gains may reflect the scale of working through 11 districts with 492 primary health facilities. ${ }^{4} 83536$ A data quality intervention in KwaZuluNatal province, South Africa, which included trainings, monthly data meetings and external data quality audits across 78 facilities improved completeness of six data elements from $26 \%$ to $64 \%$ and the agreement between facility records and reports (data accuracy) from $37 \%$ to $65 \%{ }^{6}$ A province-wide data quality intervention in Sofala, Mozambique, for 26 facilities included regular districtlevel review meetings for health workers and managers, data dashboards for tracking trends and rankings, human resource optimisation models and equipment purchase and maintenance. The summary measure used to evaluate data quality improvement, concordance, improved from $56 \%$ to $88 \%$ during the intervention period. ${ }^{4}$ The introduction of an electronic medical record to support data quality improvement in 27 facilities across Kenya recorded a decline in missing data from $31 \%$ to $13 \%$ for 24 data elements, with a mean concordance score increasing across facilities by 1.79 (95\% CI 0.25 to 3.33$){ }^{36}$

While our findings align with previous studies for increased completeness, timeliness and accuracy, our study reviewed additional WHO metrics to give a more comprehensive picture of the dimensions of data quality. This study also provided an opportunity to reflect on the relative usefulness of assessing all WHO data quality metrics to understand the quality of routine data in a given context. Sharp increases in service uptake due to health campaigns or targeted health projects make the assessment of moderate outliers and consistency over time less insightful about data quality, especially in the context of urgent efforts towards achieving universal health coverage. Our study found that, other than early postpartum-postnatal care within 3 days of birth, there were no instances of agreement between the facilitybased routine data and external household surveys. However, an emerging body of criterion validity studies have demonstrated mixed results in the ability of women to recall facility-based pregnancy-related and childbirthrelated events in household surveys. ${ }^{54-58}$ More research is needed on how to reconcile health facility and household survey data, while also reconsidering the emphasis on the household surveys as the reference standard.

The data quality metric to assess the consistency between related data elements provided useful insights in addition to the completeness and accuracy regularly reported in the literature. Assessing the data relationship for a service provision compared with a contact indicator (eg, antenatal care anaemia testing compared with antenatal care first visits) allows for discussion on whether observed discrepancies are due to low service uptake or poor reporting, an important consideration given the emphasis on improving quality of care and understanding effective coverage. ${ }^{59}$ Further, assessing data relationships that require concurrent tallying of services/information across data sources (eg, facility attendance=inpatient+outpatient; normal delivery+caesarean delivery+assisted delivery=live births+still births), provides useful insight about whether a facility is paying attention to the internal consistency of their data within and across facility documentation. Focus on accuracy of facility reporting, a more common metric assessed in the peer-reviewed literature (referred to as data accuracy or concordance), is an important data quality metric as subnational, national and global-level monitoring cannot take place effectively without the confidence that the facilities have summarised and tallied the data as intended. However, this focus on the accuracy of facility reporting up through the different levels of the health system do not require that these data be internally consistent with other data.

Our study had limitations. Without a concurrent comparison group, our before-and-after analyses cannot eliminate the effects of concurrent events and activities on data quality metrics. It is possible that other activities contributed to the observed data quality improvements. Given the high burden of maternal and neonatal mortality, the Gombe State Primary Health Care Development Agency spearheaded an initiative to improve maternal and neonatal services with the aim of having one fully functional primary health facility in each of its 114 wards. During the intervention period, 57 facilities (12\% of the 492 maternity facilities) received support including facility-level quality improvement support as well as community-based outreach and education to increase uptake of services. Facility-level activities included support on data quality to monitor trends in services provided and the provision of computers and facility registers. Additionally, similar to other data quality assessments, we did not validate the data through direct clinical observations ${ }^{461012326061}$ nor did we compare the paper-based monthly summary reports to their electronic versions in DHIS2. ${ }^{623762-64}$ Despite close attention to quality control, the facility-level and household-level surveys might still be susceptible to errors in data recording, including incorrectly tallying the number of events in the original facility registers for comparison with data in DHIS2. 
Improving the quality of routine facility data is essential for local and national evidence-based monitoring of universal health coverage. We found that an integrated district-focused data quality intervention was associated with increases across most WHO data quality metrics for routine facility-based data. Future initiatives should aim to incorporate national-level and higher subnationallevels of the health system to determine scalability and sustainability of integrated data quality interventions in the long-term.

Acknowledgements The authors wish to acknowledge the leadership of the Gombe State Primary Health Care Development Agency throughout the development and implementation of the data quality intervention. We also appreciate the cooperation of and partnership with the LGA M\&E officers, MNCH coordinators, and Gombe State implementing partners to improve the quality of routine maternal and newborn health data in Gombe State. We are grateful to the women who participated in the household surveys and to the team who conducted the facility and household data collection. Finally, we are grateful to the facility teams responsible for providing and documenting their care for women and newborns.

Contributors $A A B$ and TM conceived and designed the study. $A A B$ and EA designed the analyses. $A A B$ carried out the analyses and composed the initial draft. JS, TM, EA, NU, AA, HF reviewed the early drafts. All authors approved the final draft.

Funding This work was supported by the Bill \& Melinda Gates Foundation (OPP1149259). The funder of this study had no role in the study's design or conduct, data collection, analysis or interpretation of results, writing of the paper or decision to submit for publication.

Competing interests HF and AA are members of the Gombe State Primary Health Care Development Agency. The authors declare no other competing interests.

Patient consent for publication Not required.

Ethics approval Study approvals were obtained from the London School of Hygiene \& Tropical Medicine (reference 14091) and the Health Research Ethics Committees for Nigeria (reference NHREC/01/01/2007) and Gombe State (reference ADM/S/658/Nol. II/66).

Provenance and peer review Not commissioned; externally peer reviewed.

Data availability statement Data are available in a public, open access repository. Data for this study are available from the London School of Hygiene \& Tropical Medicine public repository: http://datacompass.Ishtm.ac.uk/229/.

Supplemental material This content has been supplied by the author(s). It has not been vetted by BMJ Publishing Group Limited (BMJ) and may not have been peer-reviewed. Any opinions or recommendations discussed are solely those of the author(s) and are not endorsed by BMJ. BMJ disclaims all liability and responsibility arising from any reliance placed on the content. Where the content includes any translated material, BMJ does not warrant the accuracy and reliability of the translations (including but not limited to local regulations, clinical guidelines, terminology, drug names and drug dosages), and is not responsible for any error and/or omissions arising from translation and adaptation or otherwise.

Open access This is an open access article distributed in accordance with the Creative Commons Attribution 4.0 Unported (CC BY 4.0) license, which permits others to copy, redistribute, remix, transform and build upon this work for any purpose, provided the original work is properly cited, a link to the licence is given, and indication of whether changes were made. See: https://creativecommons.org/ licenses/by/4.0\%.

ORCID iD

Antoinette Alas Bhattacharya http://orcid.org/0000-0001-5400-9383

\section{REFERENCES}

1 Nutley T. Improving data use in decision making: an intervention to strengthen health systems. Chapel Hill, North Carolina: MEASURE Evaluation, 2012.

2 AbouZahr C, Boerma T. Health information systems: the foundations of public health. Bull World Health Organ 2005;83:578-83.
3 Nisingizwe MP, lyer HS, Gashayija M, et al. Toward utilization of data for program management and evaluation: quality assessment of five years of health management information system data in Rwanda. Glob Health Action 2014;7:25829.

4 Wagenaar BH, Gimbel S, Hoek R, et al. Effects of a health information system data quality intervention on concordance in Mozambique: time-series analyses from 2009-2012. Popul Health Metr 2015;13:9.

5 Chilundo B, Sundby J, Aanestad M. Analysing the quality of routine malaria data in Mozambique. Malar $J$ 2004;3:3

6 Mphatswe W, Mate KS, Bennett B, et al. Improving public health information: a data quality intervention in KwaZulu-Natal, South Africa. Bull World Health Organ 2012;90:176-82.

7 Mutale W, Chintu N, Amoroso C, et al. Improving health information systems for decision making across five sub-Saharan African countries: implementation strategies from the African health Initiative. BMC Health Serv Res 2013;13:S9.

8 Braa J, Heywood A, Sahay S. Improving quality and use of data through data-use workshops: Zanzibar, United Republic of Tanzania. Bull World Health Organ 2012;90:379-84.

9 Maïga A, Jiwani SS, Mutua MK, et al. Generating statistics from health facility data: the state of routine health information systems in eastern and southern Africa. BMJ Glob Health 2019;4:e001849.

10 Bosch-Capblanch X, Ronveaux O, Doyle V, et al. Accuracy and quality of immunization information systems in forty-one low income countries. Trop Med Int Health 2009;14:2-10.

11 Ronveaux O, Rickert D, Hadler S, et al. The immunization data quality audit: verifying the quality and consistency of immunization monitoring systems. Bull World Health Organ 2005;83:503-10.

12 Mate KS, Bennett B, Mphatswe W, et al. Challenges for routine health system data management in a large public programme to prevent mother-to-child HIV transmission in South Africa. PLoS One 2009;4:e5483.

13 Maina I, Wanjala P, Soti D, et al. Using health-facility data to assess subnational coverage of maternal and child health indicators, Kenya. Bull World Health Organ 2017;95:683-94.

14 Belay H, Lippeveld T. Inventory of prism framework and tools: application of prism tools and interventions for strengthening routine health information system performance. Working paper series NO: WP-13-138. Chapel Hill, North Carolina: MEASURE Evaluation, 2013.

15 Hotchkiss DR, Aqil A, Lippeveld T, et al. Evaluation of the performance of routine information system management (PRISM) framework: evidence from Uganda. BMC Health Serv Res 2010;10:188.

16 Aqil A, Avila JE, Mejia LSP, et al. Guanajuato SINAIS assessment. technical report no: SR-10-60. Chapel Hill, North Carolina: MEASURE Evaluation, 2010.

17 Lippeveld T, Sauerborn R, Bodart C, et al. Design and implementation of health information systems. Geneva: World Health Organization, 2000.

18 Ledikwe JH, Grignon J, Lebelonyane R, et al. Improving the quality of health information: a qualitative assessment of data management and reporting systems in Botswana. Health Res Policy Syst 2014;12:7.

19 Admon AJ, Bazile J, Makungwa $\mathrm{H}$, et al. Assessing and improving data quality from community health workers: a successful intervention in Neno, Malawi. Public Health Action 2013;3:56-9.

20 Gimbel S, Mwanza M, Nisingizwe MP, et al. Improving data quality across 3 sub-Saharan African countries using the consolidated framework for implementation research (CFIR): results from the African health Initiative. BMC Health Serv Res 2017;17:828.

21 Karemere JN. Centers of excellence in monitoring and evaluation: an approach to improving data quality for effective decision making in the Democratic Republic of the Congo. Am J Trop Med Hyg 2017; $97: 284-5$.

22 Orji B, Olisaekee G, Udenze O, et al. Improving quality of data to advance malaria in pregnancy indicator coverage in Ebonyi state, Nigeria. Am J Trop Med Hyg 2017;97:335.

23 Westercamp N, Staedke S, Hutchinson E, et al. Effectiveness and sustainability of a collaborative improvement method to increase the quality of routine malaria surveillance data in Kayunga district, Uganda. Am J Trop Med Hyg 2017;97:31.

24 Kamadjeu RM, Tapang EM, Moluh RN. Designing and implementing an electronic health record system in primary care practice in subSaharan Africa: a case study from Cameroon. Inform Prim Care 2005;13:179-86.

25 Barrington J, Wereko-Brobby O, Ward P, et al. SMS for life: a pilot project to improve anti-malarial drug supply management in rural Tanzania using standard technology. Malar J 2010;9:298. 
26 Ledikwe JH, Reason LL, Burnett SM, et al. Establishing a health information workforce: innovation for low- and middle-income countries. Hum Resour Health 2013;11:35.

27 Kiberu VM, Matovu JKB, Makumbi F, et al. Strengthening districtbased health reporting through the district health management information software system: the Ugandan experience. BMC Med Inform Decis Mak 2014;14:40.

28 Ye M, N'Gbichi JM, Franchard T, et al. Malaria data quality and use in selected centers of excellence in Madagascar: results from a crosssectional baseline survey. Am J Trop Med Hyg 2019;101:118-9.

29 Sherr K, Cuembelo F, Michel C, et al. Strengthening integrated primary health care in Sofala, Mozambique. BMC Health Serv Res 2013;13:S4.

30 Dodo M, Badolo O, Nebie S, et al. Contribution of quarterly malaria data review and validation to data quality and malaria services improvement. Am J Trop Med Hyg 2018;99:118

31 Tuti T, Bitok M, Malla L, et al. Improving documentation of clinical care within a clinical information network: an essential initial step in efforts to understand and improve care in Kenyan hospitals. BMJ Glob Health 2016;1:e000028.

32 Puttkammer N, Baseman JG, Devine EB, et al. An assessment of data quality in a multi-site electronic medical record system in Haiti. Int J Med Inform 2016;86:104-16.

33 Maokola W, Willey BA, Shirima K, et al. Enhancing the routine health information system in rural southern Tanzania: successes, challenges and lessons learned. Trop Med Int Health 2011;16:721-30.

34 Evaluation M. Routine health information systems: a curriculum on basic concepts and practices. Chapel Hill, North Carolina: MEASURE Evaluation, 2017

35 Gething PW, Noor AM, Gikandi PW, et al. Improving imperfect data from health management information systems in Africa using spacetime geostatistics. PLoS Med 2006;3:e271.

36 Muthee V, Bochner AF, Osterman A, et al. The impact of routine data quality assessments on electronic medical record data quality in Kenya. PLoS One 2018;13:e0195362.

37 Gimbel S, Micek M, Lambdin B, et al. An assessment of routine primary care health information system data quality in Sofala Province, Mozambique. Popul Health Metr 2011;9:12.

38 Izugbara CO, Wekesah FM, Adedini SA. Maternal health in Nigeria: a situation update. Nairobi, Kenya: African Population and Health Research Center, 2016.

39 National Bureau of Statistics and United Nations Children's Fund. Multiple indicator cluster survey 2016-17: survey findings report. Abuja, Nigeria: National Bureau of Statistics and United Nations Children's Fund, 2017.

40 Gombe State Government. History of Gombe state, Nigeria, 2018. Available: http://gombestate.gov.ng/history-2/ [Accessed 9 Sep 2019].

41 National Population Commission [Nigeria] and ICF International. Nigeria demographic and health survey 2018, final report. Abuja, Nigeria, and Rockville, Maryland, USA: NPC/Nigeria and ICF International, 2019.

42 Nigeria District Health Information System version 2. Nigeria health management information system, version 2013: Gombe state facility deliveries, by facility type, January-December 2018. Abuja: Nigeria District Health Information System version 2, 2019.

43 Federal Government of Nigeria. Integrating primary healthcare governance in Nigeria: implementation manual, 2013.

44 National Primary Health Care Development Agency. Integrating primary health care governance in Nigeria (primary health care under one roof. Abuja: NPHCDA, 2013.

45 WHO. Data quality review: a toolkit for facility data quality assessment. Geneva: World Health Organization, 2017.

46 Lippeveld T. Routine health facility and community information systems: creating an information use culture. Glob Health Sci Pract 2017;5:338-40.
47 Marchant T. Change in maternal and newborn health care. interactions between families and frontline workers - their frequency, quality, and equity - and coverage of interventions for mothers and newborns. Report from Gombe state, Nigeria, 2012-2015 ideas project report. London School of Hygiene and Tropical Medicine, 2016.

48 WHO Service availability and readiness assessment (SARA): an annual monitoring system for service delivery. reference manual, version 2.2. Geneva: World Health Organization, 2015.

49 Bottomley C, Kirby MJ, Lindsay SW, et al. Can the buck always be passed to the highest level of clustering? BMC Med Res Methodol 2016;16:29.

50 Bhattacharya AA, Umar N, Audu A, et al. Quality of routine facility data for monitoring priority maternal and newborn indicators in DHIS2: a case study from Gombe state, Nigeria. PLoS One 2019;14:e0211265.

51 Bryce J, Requejo J, Countdown to 2015 Working Group. Countdown to 2015: tracking progress in maternal, newborn, and child survival. The 2008 report. New York: UNICEF, 2008.

52 WHO. Countdown to 2015: a decade of tracking progress for maternal, newborn and child survival, final report. Geneva: World Health Organization, 2015

53 Wagenaar BH, Hirschhorn LR, Henley C, et al. Data-driven quality improvement in low-and middle-income country health systems: lessons from seven years of implementation experience across Mozambique, Rwanda, and Zambia. BMC Health Serv Res 2017;17:830.

54 Stanton CK, Rawlins B, Drake M, et al. Measuring coverage in $\mathrm{MNCH}$ : testing the validity of women's self-report of key maternal and newborn health interventions during the Peripartum period in Mozambique. PLoS One 2013;8:e60694.

55 Blanc AK, Warren C, McCarthy KJ, et al. Assessing the validity of indicators of the quality of maternal and newborn health care in Kenya. J Glob Health 2016;6:010405.

56 Blanc AK, Diaz C, McCarthy KJ, et al. Measuring progress in maternal and newborn health care in Mexico: validating indicators of health system contact and quality of care. BMC Pregnancy Childbirth 2016;16:255.

57 McCarthy KJ, Blanc AK, Warren CE, et al. Women's recall of maternal and newborn interventions received in the postnatal period: a validity study in Kenya and Swaziland. $J$ Glob Health 2018;8:010605.

58 Bhattacharya AA, Allen E, Umar N, et al. Monitoring childbirth care in primary health facilities: a validity study in Gombe state, northeastern Nigeria. J Glob Health 2019;9:020411.

59 Amouzou A, Leslie HH, Ram M, et al. Advances in the measurement of coverage for RMNCH and nutrition: from contact to effective coverage. BMJ Glob Health 2019;4:e001297.

60 Glèlè Ahanhanzo Y, Ouendo E-M, Kpozèhouen A, et al. Data quality assessment in the routine health information system: an application of the lot quality assurance sampling in Benin. Health Policy Plan 2015;30:837-43.

61 Makombe SD, Hochgesang M, Jahn A, et al. Assessing the quality of data aggregated by antiretroviral treatment clinics in Malawi. Bull World Health Organ 2008;86:310-4.

62 Nicol E, Dudley L, Bradshaw D. Assessing the quality of routine data for the prevention of mother-to-child transmission of HIV: an analytical observational study in two health districts with high HIV prevalence in South Africa. Int J Med Inform 2016;95:60-70.

63 Mlambo MG, Peltzer K, Koivu A. Prevention of mother-to-child transmission of HIV data completeness and accuracy assessment in health facilities of the Nkangala district. Health SA Gesondheid 2014;19:a774.

64 Omoleke SA, Tadesse MG. A pilot study of routine immunization data quality in Bunza local government area: causes and possible remedies. Pan Afr Med J 2017;27:239. 